increased levels of antinuclear antibodies versus wild-type controls. Our data also suggest that the MSH6 GV identified in lupus-prone individuals results in the accumulation of mutations at $\mathrm{A}$ in the WA hotspot (W is $\mathrm{A}$ or $\mathrm{T}$; adenine or thymine, respectively; $\mathrm{A}$ is adenine) motif. Therefore, these mutations are likely to be the result of processing of the activation induced cytidine deaminase (AID)-generated $U: G$ mispair by the MSH2/6 complex followed by error-prone DNA synthesis by DNA polymerase eta.

Conclusions Importantly, these types of mutations likely result in an overall increase in positively charged amino acids in the autoantibodies that are produced, a trait that is commonly found in anti-DNA antibodies. In summary, our results suggest that the MSH6 GV has strong potential to be associated with the development of lupus.

Funding Source(s): 5 R01 ES019179-08 ; R21 AI124055-01

The MSH6GV mice develop antinuclear antibodies at 6 months of age. +/+are WT; Mut/+are heterozygotes and Mut/Mut are homozygotes.

\section{THE IMMUNE CELL LANDSCAPE IN KIDNEYS OF LUPUS NEPHRITIS PATIENTS}

${ }^{1}$ Celine C Berthier*, ${ }^{2}$ Arnon Arazi, ${ }^{3}$ Deepak Rao, ${ }^{4}$ Anne Davidson, ${ }^{5}$ Edward Browne, ${ }^{2}$ Thomas Eisenhaure, ${ }^{6}$ Nir Hacohen, ${ }^{7}$ David J Lieb, ${ }^{4}$ Betty Diamond, ${ }^{8}$ Matthias Kretzler. ${ }^{1}$ University of Michigan; ${ }^{2}$ Broad Institute; ${ }^{3}$ Brigham and Women's Hospital/Harvard Medical School; ${ }^{4}$ Feinstein Institute for Medical Research; ${ }^{5}$ UNC-Chapel Hill; ${ }^{6}$ Harvard Medical School; ${ }^{7}$ Broad Institute of MIT and Harvard; ${ }^{8}$ University of Michigan Medical School

\subsection{6/lupus-2019-Ism.204}

Background Lupus nephritis is a potentially fatal autoimmune disease, whose current treatment is ineffective and often toxic. In 2014, the National Institute of Health (NIH), industry and non-profit organizations joined their efforts with the AMP project, whose goal is to identify new diagnostic and therapeutic targets through a better understanding of the mechanisms by which individual cell types contribute to autoimmune tissue damage.

Methods To gain insights into disease mechanisms, we analyzed kidney samples from lupus nephritis patients and healthy controls using single-cell RNA-seq. Renal biopsies from 24 LN patients and 10 pre-transplant living donors (LD) were acquired across a distributed research network using a single, uniform pipeline developed by the AMP network. In brief, biopsies were cryopreserved and shipped to a centralized processing site for tissue dissociation. A total of 3541 leukocytes and 1621 epithelial cells were sorted from LN kidney samples. 438 leukocytes and 572 epithelial cells were sorted from LD biopsies. The transcriptome of those LN single tissue-infiltrating cells were assessed using single cell RNA-seq.

Results Our analysis revealed 21 subsets of leukocytes active in disease, including multiple populations of myeloid, T, NK and $\mathrm{B}$ cells, demonstrating both pro-inflammatory and resolving responses. We found evidence of local activation of B cells correlated with an age-associated B cell signature, and of progressive stages of monocyte differentiation within the kidney. A clear interferon response was observed in most cells. Two chemokine receptors, $\mathrm{C} \times \mathrm{CR} 4$ and $\mathrm{C} \times 3 \mathrm{CR} 1$, were broadly expressed, pointing to potential therapeutic targets. Gene expression of immune cells in urine and kidney was highly correlated, suggesting urine may be a surrogate for kidney biopsies.
Conclusions Our results provide a first comprehensive view of the complex network of leukocytes active in lupus nephritis kidneys. Results from this Phase 1 study identified LN active cells and pathways that can be used to guide the development of novel therapies. Analyses at a bigger scale $(n=200 \mathrm{LN})$ in Phase 2 will allow to correlate patterns and signatures of infiltrating cells with those of intrinsic renal cells, particularly the epithelial cells that make up $90 \%$ of renal cells and that are prone to hypoxic damage and cellular stress. It will accelerate the discovery of new therapeutic targets and identification of biomarkers to guide therapeutic decisions in $\mathrm{LN}$ and integrate the treatment effect.

Funding Source(s): Funding was provided through grants from the National Institutes of Health (UH2-AR067676, UH2AR067677, UH2-AR067679, UH2-AR067681, UH2-AR067685, UH2-AR067688, UH2-AR067689, UH2-AR067690, UH2AR067691, UH2-AR067694, and UM2-AR067678).

\section{SINGLE CELL RNA EXPRESSION IN LUPUS NEPHRITIS COMPARING AFRICAN-AMERICAN AND CAUCASIAN PATIENTS IDENTIFIES DIFFERENTIAL EXPRESSION OF TYPE I INTERFERON PATHWAY}

${ }^{1}$ Andrea Fava* ${ }^{2}$ Yuji Zhang, ${ }^{3}$ Nir Hacohen, ${ }^{4}$ Arnon Arazi, ${ }^{5}$ Celine C Berthier, ${ }^{6}$ Deepak Rao, ${ }^{7}$ Michael Brenner ${ }^{8}$ David Wofsy, ${ }^{9}$ Anne Davidson, ${ }^{10}$ Matthias Kretzler, ${ }^{11}$ David Hildeman, ${ }^{12}$ E Steve Woodle, ${ }^{9}$ Betty Diamond, ${ }^{13}$ Michelle Petri. ${ }^{1}$ Johns Hopkins University; ${ }^{2}$ University of Maryland; ${ }^{3}$ Harvard Medical School; ${ }^{4}$ Broad Institute; ${ }^{5}$ University of Michigan; ${ }^{6}$ Brigham and Women's Hospital/Harvard Medical School; ' Brigham And Women's Hospital; ${ }^{8}$ Russell/ Engleman Rheumatology Research Center, University of California, San Francisco, CA, USA; ${ }^{9}$ Feinstein Institute for Medical Research; ${ }^{10}$ University of Michigan Medical School; ${ }^{11}$ University of Cincinnati Department of Pediatrics; ${ }^{12}$ University of Cincinnati; ${ }^{13}$ Johns Hopkins University School of Medicine

\subsection{6/lupus-2019-Ism.205}

Background African-American ethnicity is associated with a 3fold higher risk of developing systemic lupus erythematosus (SLE). In addition, there is an increased risk of lupus nephritis (2-fold), high-risk histological features, and resistance to treatment. This may account for the increased mortality rate compared to Caucasian patients, especially in women. In Phase One of the Accelerating Medicines Partnership (AMP) study, we used single-cell RNA sequencing on kidney biopsies from patients with active lupus nephritis to identify pathways that were differentially expressed in African-American patients.

Methods Single cell RNA sequencing was performed on renal biopsies obtained for clinical purpose for active nephritis using CEL-Seq2. Cell clusters with similar expression profile were identified using $\mathrm{t}$-distributed stochastic neighbor embedding $(\mathrm{t}-$ $\mathrm{SNE}$ ). First, the relative abundance of a cluster in AAs compared to Caucasian was determined using a logistic mixed model. Second, the differential expression profile was determined for each cell cluster and we applied Ingenuity Pathway Analysis (IPA) (QIAGEN Bioinformatics) to identify pathways of interest.

Results Samples from 13 AA and 7 Caucasian patients were obtained. Of the 3097 sequenced cell libraries, we used 2354 which passed our quality filter for a total of 30155 unique molecular identifiers. We identified 16 cell clusters including CD4, CD8, B and plasma cells, NK, myeloid cells, and tubular cells. We identified 2 cell clusters unique to African-American patients, a $\mathrm{T}$ and a $\mathrm{B}$ cell population with high expression of interferon inducible genes. We also identified that same cell populations may have differential gene expression profiles 
across ethnicity. For example, CD4 T cells in African-Americans have a higher expression of type 1 and type 2 interferon pathways. In contrast, myeloid cells have several upregulated pathways in Caucasians, including ERK/MAPK signaling.

Conclusions African-American lupus nephritis patients may have a stronger interferon pathway activation in infiltrating immune cells. Several other pathways, including ERK/MAPK, are differentially expressed in infiltrating cells based on ethnicity. These results suggest that ethnicity might predict a response to both current and upcoming treatments, paving the way for a more personalized approach to treatment in lupus nephritis. Further work in Phase 2 of AMP will confirm and extend these findings.

Funding Source(s): NIH foundation partnership with AbbVie, Biogen, Bristol-Myers Squibb, Celgene, GlaxoSmithKline, Johnson and Johnson, Lilly, Merck, Pfizer, Sanofi, Takeda, and Verily.

\section{IMPACT OF THE BIRTH MONTH IN THE DEVELOPMENT OF SYSTEMIC LUPUS ERYTHEMATOSUS}

Renan Frittoli, Roberto Marini, Lilian Costallat, Simone Appenzeller*. University of Campinas

\subsection{6/lupus-2019-Ism.206}

Background There is evidence that most individuals with Systemic Lupus Erythematosus (SLE) have been born at the end of the winter season, mainly because of the influence of the mother's exposure to sunlight during pregnancy, possibly affecting vitamin D metabolism. The objective was to evaluate the influence of the birth month in the development of SLE.

Methods We included consecutive patients with childhoodonset SLE (cSLE) (age at onset of disease 16 years) and adultonset SLE (age of onset of disease $>16$ years) from the Rheumatology outpatient unit in Brazil. The control group consisted of volunteers no history of autoimmune disease. Through the review of medical records the patient's date of birth was obtained and the patients were classified according to the months and seasons of the year in which they were born. The results were presented in a descriptive way and the statistical analysis was performed through the chi-square test. For all analyzes $\mathrm{p}<0.05$ was considered statistically significant. Results A total of 1460 subjects (760 patients and 700 controls) were included. Of the patients analyzed, $662(87.1 \%)$ were adult-onset SLE and 98 cSLE (12.89\%). The mean age of the adult SLE was 42.4 years $(\mathrm{SD} \pm 12.7)$ and cSLE was 17.8 years $(\mathrm{SD} \pm 4.4)$. The controls had a mean age of 24.5 years (SD \pm 10.1 ). Patients who were born at the end of the winter season $[n=65(8.5 \%)]$ presented a statistically significant difference in relation to the control group $[n=55(7.8 \%)]$ $(p=0.011)$. When it was considered only patients with cSLE, it was observed a significantly higher birth numbers of cSLE patients during the winter season in Brazil (June 21-September 21) when compared to the controls $(p=0.018)$, and cSLE presented presented a birth frequency in winter (35.7\%) twice as high as those born in summer (17.34\%) and spring (17.34\%). A significant difference was also observed in cSLE in the month of August (which is winter in Brazil) $(p=0.042)$, when compared to the controls. Adult SLE had no differences with the control group in any month $(\mathrm{p}>0.05)$.

Conclusions It is believed that the winter season interferes with the development of SLE, especially in cSLE. These results may reinforce the idea that climate can be a contributing factor to the development of cSLE.

Funding Source(s): CNPq

FAPESP

\section{A HIGH GENETIC RISK SCORE IS ASSOCIATED WITH EARLY DISEASE ONSET, ORGAN DAMAGE AND DECREASED SURVIVAL IN SYSTEMIC LUPUS ERYTHEMATOSUS}

\begin{abstract}
${ }^{1}$ Sarah Reid*, ${ }^{2}$ Andrei Alexsson, ${ }^{3}$ Martina Frodlund, ${ }^{2}$ Johanna Sandling, ${ }^{2}$ Karin Bolin, ${ }^{4}$ Elisabet Svenungsson, ${ }^{5}$ Andreas Jönsen, ${ }^{6}$ Christine Bengtsson, ${ }^{7}$ Iva Gunnarsson, ${ }^{5}$ Anders A Bengtsson, ${ }^{8}$ Solbritt Rantapää-Dahlqvist, ${ }^{2}$ Maija-Leena Eloranta, ${ }^{2}$ Ann-Christine Syvänen, ${ }^{3}$ Christopher Sjöwall, 'Lars Ronnblom, ${ }^{1}$ Dag Leonard. ${ }^{1}$ Uppsala university; ${ }^{2}$ Dept of Medical Sciences, Science for Life Laboratories, Rheumatology, Uppsala University; ${ }^{3}$ Linköping University; ${ }^{4}$ Karolinska Insitutet; ${ }^{5}$ Dept of Rheumatology, Skåne University Hospital; ${ }^{6}$ Christine Bengtsson; ${ }^{7}$ Dept of Medicine, Karolinska Institutet; ${ }^{8}$ Dept of Public Health and Clinical Medicine/Rheumatology, Umeå University
\end{abstract}

10.1136/lupus-2019-Ism.207

Background Systemic lupus erythematosus (SLE) is a chronic, autoimmune disease with a complex genetic etiology. Over 100 risk genes for SLE have been identified at genome-wide significance, but their overall effect on disease severity has not previously been studied. We therefore assessed the relationship between a high genetic risk score and the development of organ damage in SLE.

Methods Patients with SLE, who met 4 ACR criteria $(\mathrm{n}=1001)$, and healthy controls $(\mathrm{n}=2802)$ were genotyped using a 200K Immunochip SNP Array (Illumina). A genetic risk score (GRS) was assigned to each individual based on 57 SLE risk loci which have previously shown association $(\mathrm{p}<5 \times 10-8)$ with SLE according to Chen et al (Curr Opin Rheumatol, 2017; 29(5):423-433), weighted by their SLE susceptibility odds ratios (ORs). Clinical data was retrieved from medical charts.

Results SLE prevalence increased with increasing GRS (figure 1A) and was higher in the highest compared to the lowest GRS-quartile (OR 12.32 (9.5315.71) $\quad \mathrm{p}=7.9 \times 10-86)$. SLE onset occurred 5 years earlier in the high compared to the low quartile (figure 1B). The OR for organ damage increased with increasing GRS (figure 1C) and was significantly higher in the high compared to the low GRS-quartile (OR 1.47 $(1.062 .04) \mathrm{p}=2.0 \times 10-2)$. Moreover, patients in the high quartile had an increased prevalence of nephritis (OR 2.22 (1.503.27), $\mathrm{p}=5.9 \times 10-5)$, end-stage kidney disease (ESKD) (OR 5.58 (1.5020.79), $\mathrm{p}=1.0 \times 10-2)$ anti-dsDNA antibodies (OR 1.83 (1.192.81), $\mathrm{p}=6.1 \times 10-03)$, anti-cardiolipin-IgG (OR 2.16 (1.303.59), $\mathrm{p}=2.8 \times 10-03)$ and anti-2-glycoprotein-I (OR 1.69 (1.042.74), $\mathrm{p}=3.3 \times 10-02)$. Analysis of renal biopsy data showed that the prevalence of proliferative nephritis was significantly higher in the high, compared to the low, quartile (OR 2.42 (1.304.49), $\mathrm{p}=5.1 \times 10-03)$. Moreover, the patients in the high GRS-quartile displayed decreased survival until their first organ damage (HR 1.51 (1.042.25), $\mathrm{p}=3.7 \times 10-02)$, first cardiovascular event (HR 1.65 (1.032.64), $\mathrm{p}=2.6 \times 10$ $02)$, nephritis onset (HR $2.53(1.723 .71) \mathrm{p}=9.6 \times 10-7)$ and ESKD (6.78 (1.7826.86), $\mathrm{p}=6.5 \times 10-3)$. Lastly, OR for mortality increased with increasing GRS (figure 1D), with a 5 year decrease in overall survival in the high compared to the low quartile (HR 1.82 (1.043.19), $\mathrm{p}=2.4 \times 10-2$ ).

Conclusions A high genetic risk score is associated with earlier disease onset, increased risk of organ damage and impaired 